\title{
A Note on Fully Entangled Fraction
}

\author{
Ming-Jing Zhao ${ }^{1}$, Zong-Guo $\mathrm{Li}^{2}$, Shao-Ming Fei ${ }^{1}$, and Zhi-Xi Wang ${ }^{1}$ \\ ${ }^{1}$ School of Mathematical Sciences, Capital Normal University, Beijing 100048, China \\ ${ }^{2}$ Beijing National Laboratory for Condensed Matter Physics, \\ Institute of Physics, Chinese Academy of Sciences, Beijing 100190, China
}

\begin{abstract}
We investigate the general characters of fully entangled fraction for quantum states. The fully entangled fraction of Isotropic states and Werner states are analytically computed.
\end{abstract}




\section{INTRODUCTION}

Entanglement is a vital resource for some practical applications in quantum information processing such as quantum cryptography, quantum teleportation and quantum computation [1, 2]. One way to characterize the nonclassical property of quantum entanglement is to quantify the entanglement in terms of some measures, for example, entanglement of formation [3], concurrence [4], negativity [5] and geometric measure [6, 7]. However, in fact it is the fully entangled fraction (FEF) that is tightly related to many quantum information processing such as dense coding [8], teleportation [9], entanglement swapping [10], and quantum cryptography (Bell inequalities) [11]. For instance the fidelity of optimal teleportation is given by FEF [12 14]. Additionally, the FEF in two-qubit system acts as an index to characterize the nonlocal correlation [15] and one can never determine whether a state is entangled or not through the Dür-Cirac method [16], which is a simple and effective method for examining multiqubit entanglement, if the FEF is less than or equal to $\frac{1}{2}$. FEF also plays a significant role in deriving two bounds on the damping rates of the dissipative channel [17]. Since FEF has a clear experimental meaning, an analytic formula for FEF is of great importance. In [18] an elegant formula for FEF in two-qubit system is derived analytically by using the method of Lagrange multiplier. For high dimensional quantum states the analytical computation of FEF remains formidable and less results have been known. In [19] the upper bound of FEF has been estimated.

In this paper, we first present some properties of FEF and its relations with negativity, concurrence and geometric measure. Then we analytically solve the FEF for some classes of quantum states such as Isotropic states and Werner states.

\section{PROPERTIES OF FEF}

The FEF of a density matrix $\rho$ in $d \otimes d$ Hilbert space is defined by [13, 14]

$$
\mathcal{F}(\rho)=\max _{U}\left\langle\psi^{+}\left|U^{\dagger} \otimes I \rho U \otimes I\right| \psi^{+}\right\rangle
$$

where $U$ (resp. $I$ ) is a unitary (resp. identity) matrix, $\left|\psi^{+}\right\rangle=\frac{1}{\sqrt{d}} \sum_{k=1}^{d}|k k\rangle$ is the maximally entangled pure state. 
Any $d \otimes d$ pure state $|\psi\rangle=\sum_{i, j=1}^{d} a_{i j}|i j\rangle$ can be written in the standard Schmidt form,

$$
|\psi\rangle=\sum_{i} \lambda_{i}|i i\rangle
$$

where the Schmidt coefficients $\lambda_{i}, i=1, \cdots, d$, satisfy $0 \leq \lambda_{d} \leq \cdots \leq \lambda_{2} \leq \lambda_{1} \leq 1$ and $\sum_{i} \lambda_{i}^{2}=1$. The FEF of $|\psi\rangle$ has been given in [21],

$$
\mathcal{F}(|\psi\rangle)=\frac{1}{d}\left(\sum_{i} \lambda_{i}\right)^{2} .
$$

From Eq. (3) it can be seen that $|\psi\rangle$ is separable if and only if $\mathcal{F}(|\psi\rangle)=\frac{1}{d}$.

For pure states the FEF has direct relations with some entanglement measures. For instance, due to $\left\|(|\psi\rangle\langle\psi|)^{T_{1}}\right\|=\left(\sum_{i} \lambda_{i}\right)^{2}$, the negativity [5], $\mathcal{N}(\rho)=\frac{\left\|\rho^{T_{1}}\right\|-1}{2}$ can be expressed as $\mathcal{N}(|\psi\rangle)=\frac{d \mathcal{F}(|\psi\rangle)-1}{2}$, where $T_{1}$ stands for partial transposition with respect to the first space. The geometric measure [7] is defined by $\mathcal{E}(|\psi\rangle)=1-\Lambda_{\max }^{2}(|\psi\rangle)$, where $\Lambda_{\max }^{2}(|\psi\rangle)=$ $\sup _{|\phi\rangle \in S}|\langle\psi \mid \phi\rangle|^{2}$ and $S$ denotes the set of product states. For pure state $|\psi\rangle$ in Eq. (2), we have $\Lambda_{\max }^{2}(|\psi\rangle)=\lambda_{1}^{2}$ and $\mathcal{E}(|\psi\rangle)=1-\lambda_{1}^{2}$. From Eq. (3), we can get the relation between FEF and geometric measure: $d \Lambda_{\max }^{2} \geq \mathcal{F}$ and $\mathcal{F} \leq d(1-\mathcal{E})$.

For $d \otimes d$ mixed states $\rho=\sum_{i} p_{i}\left|\psi_{i}\right\rangle\left\langle\psi_{i}\right|, \mathcal{F}(\rho)$ has no general analytical formula. It can be shown that

$$
\mathcal{F}(\rho) \leq \sum_{i} p_{i} \mathcal{F}\left(\left|\psi_{i}\right\rangle\right)
$$

since

$$
\mathcal{F}(\rho) \leq \sum_{i} p_{i} \max _{U_{i}}\left\langle\psi^{+}\left|U_{i}^{\dagger} \otimes I\right| \psi_{i}\right\rangle\left\langle\psi_{i}\left|U_{i} \otimes I\right| \psi^{+}\right\rangle=\sum_{i} p_{i} \mathcal{F}\left(\left|\psi_{i}\right\rangle\right)
$$

From Eq. (44) and the main result in [20], we can obtain a relation between FEF and concurrence for mixed states, $\mathcal{C}(\rho) \geq \max \left\{\sqrt{\frac{2}{d(d-1)}}(d \mathcal{F}(\rho)-1), 0\right\}$. For two-qubit states, using the relation between the entanglement of formation and the concurrence, one gets the relation between the entanglement of formation and FEF presented in [12].

Most of the entanglement measures for a mixed state $\rho$ are defined in terms of all possible pure state decompositions of $\rho$ by convex roof, e.g. for concurrence $C, C(\rho) \equiv$ $\min _{\left\{p_{i},\left|\psi_{i}\right\rangle\right\}} \sum_{i} p_{i} C\left(\left|\psi_{i}\right\rangle\right)$. A question one may ask is whether the FEF of a mixed state also has such property: $\mathcal{F}(\rho) \equiv \min _{\left\{p_{i},\left|\psi_{i}\right\rangle\right\}} \sum_{i} p_{i} \mathcal{F}\left(\left|\psi_{i}\right\rangle\right)$. The answer is no. As a counterexample one may consider $2 \otimes 2$ state $\rho=\frac{1}{2}(|00\rangle\langle 00|+| 11\rangle\langle 11|)$. By direct calculation one has $\mathcal{F}(\rho)=\frac{1}{2}$. While for any other decompositions $\left\{p_{i},\left|\psi_{i}\right\rangle\right\}$ with $\left|\psi_{i}\right\rangle=\alpha_{i}|00\rangle+\beta_{i}|11\rangle$, where $\alpha_{i}, \beta_{i} \in \mathbb{C}$ and $\left|\alpha_{i}\right|^{2}+\left|\beta_{i}\right|^{2}=1, \sum_{i} p_{i} \mathcal{F}\left(\left|\psi_{i}\right\rangle\right)=\frac{1}{2}+\sum_{i} p_{i}\left|\alpha_{i} \beta_{i}\right|>\mathcal{F}(\rho)$. Here we give a condition such that the equality holds in Eq. (44). 
Theorem 1 For any $d \otimes d$ mixed state $\rho=\sum_{t=1}^{n} p_{t}\left|\psi_{t}\right\rangle\left\langle\psi_{t}\right|, n>1, \mathcal{F}(\rho)=\sum_{t} p_{t} \mathcal{F}\left(\left|\psi_{t}\right\rangle\right)$ if and only if there exist unitary transformations $U_{1}^{(t)}$ and $U_{2}^{(t)}$ such that $U_{1}^{(t)} \otimes U_{2}^{(t)}\left|\psi_{t}\right\rangle=$ $\sum_{j} a_{j}^{(t)}|j j\rangle$ with $a_{j}^{(t)} \geq 0$ and $U_{1}^{(s) \dagger} U_{2}^{(s) *}=e^{i \theta_{s t}} U_{1}^{(t) \dagger} U_{2}^{(t) *}, 1 \leq s, t \leq n, 0 \leq \theta_{s t} \leq 2 \pi$. For such state, $\mathcal{F}(\rho)=\frac{1}{d} \sum_{t} p_{t}\left(\sum_{j} a_{j}^{(t)}\right)^{2}$.

Proof. We only need to prove the case $n=2$. The cases $n \geq 3$ can be similarly proved.

Assume $\rho=p_{1}\left|\psi_{1}\right\rangle\left\langle\psi_{1}\left|+p_{2}\right| \psi_{2}\right\rangle\left\langle\psi_{2}\right|$. By Schmidt decomposition, there exist unitary matrices $U_{1}^{(1)}, U_{2}^{(1)}, U_{1}^{(2)}, U_{2}^{(2)}$ such that $\left|\tilde{\psi}_{i}\right\rangle=U_{1}^{(i)} \otimes U_{2}^{(i)}\left|\psi_{i}\right\rangle=\sum_{j} a_{j}^{(i)}|j j\rangle$ with $a_{j}^{(i)} \geq 0$, $i=1,2$. We have

$$
\begin{aligned}
\mathcal{F}(\rho)= & \max _{V}\left(p_{1}\left\langle\psi^{+}\left|V^{\dagger} U_{1}^{(1) \dagger} \otimes U_{2}^{(1) \dagger}\right| \tilde{\psi}_{1}\right\rangle\left\langle\tilde{\psi}_{1}\left|U_{1}^{(1)} V \otimes U_{2}^{(1)}\right| \psi^{+}\right\rangle\right. \\
& \left.+p_{2}\left\langle\psi^{+}\left|V^{\dagger} U_{1}^{(2) \dagger} \otimes U_{2}^{(1) \dagger}\right| \tilde{\psi}_{2}\right\rangle\left\langle\tilde{\psi}_{2}\left|U_{1}^{(2)} V \otimes U_{2}^{(2)}\right| \psi^{+}\right\rangle\right) .
\end{aligned}
$$

Therefore $\mathcal{F}(\rho)=p_{1} \mathcal{F}\left(\left|\psi_{1}\right\rangle\right)+p_{2} \mathcal{F}\left(\left|\psi_{2}\right\rangle\right)$ if and only if there exists unitary matrix $V$ such that

$$
\begin{aligned}
\mathcal{F}\left(\left|\psi_{1}\right\rangle\right) & =\mathcal{F}\left(\left|\tilde{\psi}_{1}\right\rangle\right) \\
& =\left\langle\psi^{+}\left|V^{\dagger} U_{1}^{(1) \dagger} \otimes U_{2}^{(1) \dagger}\right| \tilde{\psi}_{1}\right\rangle\left\langle\tilde{\psi}_{1}\left|U_{1}^{(1)} V \otimes U_{2}^{(1)}\right| \psi^{+}\right\rangle \\
& =\operatorname{tr}\left(V^{\dagger} U_{1}^{(1) \dagger} \otimes U_{2}^{(1) \dagger}\left|\tilde{\psi}_{1}\right\rangle\left\langle\tilde{\psi}_{1}\right| U_{1}^{(1)} V \otimes U_{2}^{(1)} P_{+}\right) \\
& =\operatorname{tr}\left(U_{2}^{(1) *} V^{\dagger} U_{1}^{(1) \dagger} \otimes I\left|\tilde{\psi}_{1}\right\rangle\left\langle\tilde{\psi}_{1}\right| U_{1}^{(1)} V U_{2}^{(1) T} \otimes I P_{+}\right) \\
& =\left|\left\langle\tilde{\psi}_{1}\left|U_{1}^{(1)} V U_{2}^{(1) T} \otimes I\right| \psi^{+}\right\rangle\right|^{2}
\end{aligned}
$$

where $P_{+}=\left|\psi^{+}\right\rangle\left\langle\psi^{+}\right|$and $A \otimes I P_{+}=I \otimes A^{T} P_{+}$. Furthermore, $\mathcal{F}\left(\left|\psi_{2}\right\rangle\right)=\mathcal{F}\left(\left|\tilde{\psi}_{2}\right\rangle\right)=$ $\left|\left\langle\tilde{\psi}_{2}\left|U_{1}^{(2)} V U_{2}^{(2) T} \otimes I\right| \psi^{+}\right\rangle\right|^{2}$. On the other hand, $\mathcal{F}\left(\left|\tilde{\psi}_{1}\right\rangle\right)=\frac{1}{d}\left(\sum_{j} a_{j}^{(1)}\right)^{2}$ and $\mathcal{F}\left(\left|\tilde{\psi}_{2}\right\rangle\right)=$ $\frac{1}{d}\left(\sum_{j} a_{j}^{(2)}\right)^{2} . \mathcal{F}\left(\left|\psi_{1}\right\rangle\right)$ reaches maximum when $U_{1}^{(1)} V U_{2}^{(1) T}=e^{i \theta_{1}} I$, i.e. $U_{1}^{(1) \dagger} U_{2}^{(1) *}=e^{-i \theta_{1}} V$. Similarly, we have $U_{1}^{(2) \dagger} U_{2}^{(2) *}=e^{-i \theta_{2}} V$ and $U_{1}^{(1) \dagger} U_{2}^{(1) *}=e^{i\left(\theta_{2}-\theta_{1}\right)} U_{1}^{(2) \dagger} U_{2}^{(2) *}$. The value of FEF can be obtained from Eq. (3) .

Theorem 1 gives the condition that FEF fulfills the convex roof measure. Besides if one interprets FEF of a state $\rho$ as the distance between $\rho$ and maximally entangled states, then the larger FEF is, the closer they are. Although there are infinite maximally entangled states, the one $U \otimes I\left|\psi^{+}\right\rangle$which reaches the maximum of Eq. (1) is the closest maximally entangled state to $\rho$. The theorem 1 also tells us when the closest maximally entangled state to two different pure states are the same. As an example, we consider mixed state $\rho=\sum_{i=1}^{d} p_{i}|i, \sigma(i)\rangle\langle i, \sigma(i)|$, where $\sigma$ denotes the permutation of $(1,2, \cdots, d)$. For this state, theorem applies and we have $\mathcal{F}(\rho)=\sum_{i=1}^{d} p_{i} \mathcal{F}\left(\left|\psi_{i}\right\rangle\right)=\frac{1}{d}$ with $\left|\psi_{i}\right\rangle=|i, \sigma(i)\rangle$. The distance 
between $\left|\psi_{i}\right\rangle$ and maximally entangled states is $\frac{1}{d}$. The closest maximally entangled state to $\left|\psi_{i}\right\rangle$ is $\left|\psi_{0}\right\rangle=\frac{1}{\sqrt{d}} \sum_{i=1}^{d}|i, \sigma(i)\rangle:\left|\left\langle\psi_{i} \mid \psi_{0}\right\rangle\right|^{2}=\frac{1}{d}, i=1, \cdots, d$.

From Eq. (3) and Eq. (4) one can obtain that for any $d \otimes d$ mixed state $\rho$, if $\rho$ is separable, then $\mathcal{F}(\rho) \leq \frac{1}{d}$. Moreover

Theorem 2 For any $d \otimes d$ mixed state $\rho, \frac{1}{d^{2}} \leq \mathcal{F}(\rho) \leq 1 . \mathcal{F}(\rho)=1$ if and only if $\rho$ is a maximally entangled pure state. $\mathcal{F}(\rho)=\frac{1}{d^{2}}$ if and only if $\rho$ is the maximally mixed state, i.e. $\rho=\frac{1}{d^{2}} I$.

Proof. For any $d \otimes d$ mixed state $\rho$, we assume $\rho=\sum_{i=1}^{d^{2}} \lambda_{i}\left|\phi_{i}\right\rangle\left\langle\phi_{i}\right|$ is the spectrum decomposition such that $\sum_{i=1}^{d^{2}} \lambda_{i}=1,0 \leq \lambda_{i} \leq 1$ and $\left\{\left|\phi_{i}\right\rangle\right\}_{i=1}^{d^{2}}$ are normalized orthogonal eigenvectors in $d \otimes d$ Hilbert space. Then $\mathcal{F}(\rho)=\max _{U}\left\langle\psi^{+}\left|U^{\dagger} \otimes I \rho U \otimes I\right| \psi^{+}\right\rangle=$ $\max _{U} \sum_{i} \lambda_{i}\left\langle\psi^{+}\left|U^{\dagger} \otimes I\right| \phi_{i}\right\rangle\left\langle\phi_{i}|U \otimes I| \psi^{+}\right\rangle$. Set $a_{i}=\left\langle\psi^{+}\left|U^{\dagger} \otimes I\right| \phi_{i}\right\rangle\left\langle\phi_{i}|U \otimes I| \psi^{+}\right\rangle$, which satisfies $0 \leq a_{i} \leq 1$ and $\sum_{i=1}^{d^{2}} a_{i}=1$ due to the completeness of the eigenvectors $\left\{\left|\phi_{i}\right\rangle\right\}$. $\sum_{i=1}^{d^{2}} \lambda_{i} a_{i} \leq \sum_{i=1}^{d^{2}} \lambda_{i}=1$ becomes an equality if and only if there are only one nonzero coefficient, say, $a_{i}=1$ and one nonzero coefficient $\lambda_{i}=1$. Therefore $\mathcal{F}(\rho)=1$ if and only if $\rho$ is maximally entangled pure state.

On the other hand, the minimum of the function $g\left(\lambda_{i}, a_{i}\right)=\sum_{i=1}^{d^{2}} \lambda_{i} a_{i}$ is $\frac{1}{d^{2}}$ by Lagrange multiplier. It reaches its minimum if and only if $\lambda_{i}=a_{i}=\frac{1}{d^{2}}$ for $i=1, \cdots, d^{2}$. This gives rise to $\rho=\frac{1}{d^{2}} I$.

Similar to the proof above, here one can also obtain the range of geometric measure for mixed states.

Corollary 3 For any $d \otimes d$ mixed state $\rho$, it satisfies $0 \leq \mathcal{E}(\rho) \leq \frac{d-1}{d}$. $\mathcal{E}(\rho)=0$ if and only if $\rho$ is a separable state. $\mathcal{E}(\rho)=\frac{d-1}{d}$ if and only if $\rho$ is a maximally entangled pure state.

We have studied some properties related to the FEF. Before we compute analytically the FEF for Isotropic states and Werner states, we investigate another property that similarly studied for entanglement of formation, negativity, concurrence, geometric measure and qsquashed entanglement [22].

Theorem 4 For two given pure states $\left|\phi_{1}\right\rangle$ and $\left|\phi_{2}\right\rangle$, the FEF of their superposition $|\psi\rangle=$ $\frac{1}{\gamma}\left(\alpha\left|\phi_{1}\right\rangle+\beta\left|\phi_{2}\right\rangle\right)$ satisfies:

$\max \left\{|| \alpha \mid \mathcal{F}^{\frac{1}{2}}\left(\left|\phi_{1}\right\rangle\right)-|\beta| \mathcal{F}^{\frac{1}{2}}\left(\left|\phi_{2}\right\rangle\right) \mid, \frac{1}{d^{2}}\right\} \leq|\gamma| \mathcal{F}^{\frac{1}{2}}(|\psi\rangle) \leq \min \left\{|\alpha| \mathcal{F}^{\frac{1}{2}}\left(\left|\phi_{1}\right\rangle\right)+|\beta| \mathcal{F}^{\frac{1}{2}}\left(\left|\phi_{2}\right\rangle\right), 1\right\}(5)$ 
Proof. By the definition of FEF we have

$$
\begin{aligned}
\mathcal{F}(|\psi\rangle) & =\frac{1}{\gamma^{2}} \max _{U}\left\langle\psi ^ { + } \left|U ^ { \dagger } \otimes I ( \alpha | \phi _ { 1 } \rangle + \beta | \phi _ { 2 } \rangle ) \left(\alpha^{*}\left\langle\phi_{1}\left|+\beta^{*}\left\langle\phi_{2}\right|\right) U \otimes I \mid \psi^{+}\right\rangle\right.\right.\right. \\
& \leq \frac{1}{\gamma^{2}}\left(|\alpha|^{2} \mathcal{F}\left(\left|\phi_{1}\right\rangle\right)+|\beta|^{2} \mathcal{F}\left(\left|\phi_{2}\right\rangle\right)+2|\alpha \beta| \sqrt{\mathcal{F}\left(\left|\phi_{1}\right\rangle\right) \mathcal{F}\left(\left|\phi_{2}\right\rangle\right)}\right) \\
& =\frac{1}{\gamma^{2}}\left(|\alpha| \mathcal{F}^{\frac{1}{2}}\left(\left|\phi_{1}\right\rangle\right)+|\beta| \mathcal{F}^{\frac{1}{2}}\left(\left|\phi_{2}\right\rangle\right)\right)^{2},
\end{aligned}
$$

which gives the right hand side of Eq. (5) .

Similarly, taking into account of $\left|\phi_{1}\right\rangle=\frac{\gamma}{\alpha}|\psi\rangle-\frac{\beta}{\alpha}\left|\phi_{2}\right\rangle$ and $\left|\phi_{2}\right\rangle=\frac{\gamma}{\beta}|\psi\rangle-\frac{\alpha}{\beta}\left|\phi_{1}\right\rangle$, one gets the left hand side of Eq. (5)

For example, let $\left|\phi_{1}\right\rangle=|00\rangle,\left|\phi_{2}\right\rangle=|11\rangle$ and $|\psi\rangle=\frac{1}{\sqrt{2}}(|00\rangle+|11\rangle)$, then FEF of $|\psi\rangle$ reaches the upper bound of Eq. (51). If we take $\left|\phi_{1}\right\rangle=\frac{1}{\sqrt{2}}(|00\rangle-|11\rangle),\left|\phi_{2}\right\rangle=|11\rangle$ and $|\psi\rangle=|00\rangle$, then FEF of $|\psi\rangle$ reaches the lower bound of Eq. (15). Eq. (15) can also be generalized to the case of superposition with more than two components: for $|\psi\rangle=$ $\frac{1}{\gamma}\left(\alpha_{1}\left|\phi_{1}\right\rangle+\cdots+\alpha_{m}\left|\phi_{m}\right\rangle\right)$, we have $\max _{i}\left\{\left|\alpha_{i}\right| \mathcal{F}^{\frac{1}{2}}\left(\left|\phi_{i}\right\rangle\right)-\sum_{j \neq i}\left|\alpha_{j}\right| \mathcal{F}^{\frac{1}{2}}\left(\left|\phi_{j}\right\rangle\right), \frac{1}{d^{2}}\right\} \leq|\gamma| \mathcal{F}^{\frac{1}{2}}(|\psi\rangle) \leq$ $\min \left\{\sum_{i}\left|\alpha_{i}\right| \mathcal{F}^{\frac{1}{2}}\left(\left|\phi_{i}\right\rangle\right), 1\right\}$.

\section{FEF FOR SOME CLASSES OF MIXED STATES}

Generally for mixed states it is rather difficult to get analytical formulae for entanglement measures and FEF. Nevertheless for some special quantum states, elegant results have been derived. For instance, for the Isotropic state, entanglement of formation [23], concurrence [24] and geometric measure [7] have been calculated explicitly. For the Werner state, concurrence [25] and geometric measure [7] have been investigated also. Now we calculate analytically FEF for such well-known mixed states.

Isotropic state Isotropic states [21] are a class of $U \otimes U^{*}$ invariant mixed states in $d \otimes d$ Hilbert space:

$$
\rho_{i s o}(f)=\frac{1-f}{d^{2}-1} I+\frac{d^{2} f-1}{d^{2}-1}\left|\psi^{+}\right\rangle\left\langle\psi^{+}\right|,
$$

with $f=\left\langle\psi^{+}\left|\rho_{\text {iso }}(f)\right| \psi^{+}\right\rangle$satisfying $0 \leq f \leq 1$. These states are shown to be separable if and only if they are PPT, i.e. $f \leq \frac{1}{d}$. They can be distilled if they are entangled, which means $f>\frac{1}{d}[21]$.

By definition, the FEF is given by

$$
\mathcal{F}\left(\rho_{\text {iso }}(f)\right)=\frac{1-f}{d^{2}-1}+\max _{U} \frac{d^{2} f-1}{d^{2}-1}\left|\left\langle\psi^{+}|U \otimes I| \psi^{+}\right\rangle\right|^{2}=\frac{1-f}{d^{2}-1}+\max _{U} \frac{d^{2} f-1}{d^{2}-1}\left|\frac{1}{d} \operatorname{tr} U\right|^{2} .
$$


If $\frac{d^{2} f-1}{d^{2}-1}>0$, i.e. $f>\frac{1}{d^{2}}$, we have $\mathcal{F}\left(\rho_{i s o}(f)\right)=\frac{1-f}{d^{2}-1}+\frac{d^{2} f-1}{d^{2}-1}=f$. The maximum is attained by choosing $U=I$. If $\frac{d^{2} f-1}{d^{2}-1}<0$, i.e. $f<\frac{1}{d^{2}}$, we get $\mathcal{F}\left(\rho_{i s o}(f)\right)=\frac{1-f}{d^{2}-1}+\frac{d^{2} f-1}{d^{2}-1} \min _{U}\left|\frac{1}{d} \operatorname{tr} U\right|^{2} \leq$ $\frac{1-f}{d^{2}-1}$. In fact, if we choose $U=\sum_{i \neq j}|i\rangle\langle j|$, then the inequality becomes an equality. If $\frac{d^{2} f-1}{d^{2}-1}=0$, i.e. $f=\frac{1}{d^{2}}$, we have $\mathcal{F}\left(\rho_{\text {iso }}(f)\right)=\frac{1}{d^{2}}$. Therefore we get the FEF for Isotropic states:

$$
\mathcal{F}\left(\rho_{\text {iso }}(f)\right)=\left\{\begin{array}{cl}
f, & \frac{1}{d^{2}} \leq f \leq 1 \\
\frac{1-f}{d^{2}-1}, & 0 \leq f<\frac{1}{d^{2}}
\end{array}\right.
$$

According to [13], the fidelity $\mathbf{f}_{\max }$ of optimal teleportation via state $\rho$ attainable by means of trace-preserving local quantum operations and classical communication (LOCC) is equal to $\mathbf{f}_{\max }(\rho)=\frac{\mathcal{F}(\rho) d+1}{d+1}$. If $\mathcal{F}(\rho)>\frac{1}{d}$, then state $\rho$ is said to be useful for teleportation. Hence all entangled Isotropic states are useful in quantum teleportation.

Werner state Werner states [26] are a class of $U \otimes U$ invariant mixed states in $d \otimes d$ Hilbert space:

$$
\rho_{w e r}(f)=\frac{d-f}{d^{3}-d} I+\frac{d f-1}{d^{3}-d} V
$$

where $V=\sum_{i, j=1}^{d}|i j\rangle\langle j i|$ and $f=\left\langle\psi^{+}\left|\rho_{w e r}(f)\right| \psi^{+}\right\rangle,-1 \leq f \leq 1$. These states are shown to be separable if and only if they are $\operatorname{PPT}(f \geq 0)$.

The FEF of Werner state is given by

$$
\begin{aligned}
\mathcal{F}\left(\rho_{\text {wer }}(f)\right) & =\frac{d-f}{d^{3}-d}+\max _{U} \frac{d f-1}{d^{3}-d}\left|\left\langle\psi^{+}\left|U^{\dagger} \otimes I V U \otimes I\right| \psi^{+}\right\rangle\right| \\
& =\frac{d-f}{d^{3}-d}+\max _{U} \frac{d f-1}{d^{4}-d^{2}} \sum_{k l}\left\langle k\left|U^{\dagger}\right| l\right\rangle\langle k|U| l\rangle \\
& =\frac{d-f}{d^{3}-d}+\max _{U} \frac{d f-1}{d^{4}-d^{2}} \operatorname{tr}\left(U U^{*}\right) .
\end{aligned}
$$

i) If $d f-1>0$, since $U U^{*}$ is unitary,

$$
\mathcal{F}\left(\rho_{\text {wer }}(f)\right)=\frac{d-f}{d^{3}-d}+\frac{d f-1}{d^{3}-d}=\frac{f+1}{d(d+1)},
$$

which corresponds to the case $U=I$.

ii) If $d f-1<0$ and $d$ is even, we get

$$
\mathcal{F}\left(\rho_{w e r}(f)\right)=\frac{d-f}{d^{3}-d}-\frac{d f-1}{d^{3}-d}=\frac{1-f}{d(d-1)},
$$

which can be attained by choosing $U=A_{2 \times 2} \otimes I_{\frac{d}{2} \times \frac{d}{2}}$ with $A_{2 \times 2}=\left(\begin{array}{cc}0 & 1 \\ -1 & 0\end{array}\right)$. 
iii) For the case of $d f-1<0$ and $d$ is odd, one has

$$
\mathcal{F}\left(\rho_{w e r}(f)\right)=\frac{d-f}{d^{3}-d}+\frac{d f-1}{d^{3}-d} \times \frac{-d+2}{d}=\frac{d^{2}-d^{2} f+d f+d-2}{d^{2}\left(d^{2}-1\right)} .
$$

vi) If $d f-1=0$, i.e. $f=\frac{1}{d}, \mathcal{F}\left(\rho_{w e r}(f)\right)=\frac{1}{d^{2}}$.

Therefore we get the FEF for Werner states:

$$
\mathcal{F}\left(\rho_{\text {wer }}(f)\right)= \begin{cases}\frac{f+1}{d(d+1)}, & \frac{1}{d} \leq f \leq 1 \\ \frac{1-f}{d(d-1)}, & -1 \leq f<\frac{1}{d}\end{cases}
$$

if $\mathrm{d}$ is even; and

$$
\mathcal{F}\left(\rho_{\text {wer }}(f)\right)=\left\{\begin{array}{cc}
\frac{f+1}{d(d+1)}, & \frac{1}{d} \leq f \leq 1 \\
\frac{d^{2}-d^{2} f+d f+d-2}{d^{2}\left(d^{2}-1\right)}, & -1 \leq f<\frac{1}{d} .
\end{array}\right.
$$

if $\mathrm{d}$ is odd. Hence this formula tells us there exist entangled Werner states which are not useful for teleportation.

\section{CONCLUSIONS}

We have explored some characters of FEF and analytically computed the FEF of several well-known classes of quantum mixed states. These results complement previous ones in this subject and may give rise to new application to the quantum information processing.

Acknowledgments This work is supported by the NSF of China (Grant Nos. 10875081,10871227), the NSF of Beijing (Grant No. 1092008), KZ200810028013 and PHR201007107.

[1] Bennett C H and DiVincenzo D P 2000 Nature (London) 404, 247

[2] Nielsen M A and Chuang I L 2000 Quantum Computation and Quantum Information (Cambridge University Press, Cambridge)

[3] Bennett C H, Bernstein H J, Popescu S and Schumacher B 1996 Phys. Rev. A 53, 2046

[4] Uhlmann A 2000 Phys. Rev. A 62, 032307; Rungta P, Bužek V, Caves C M, Hillery M and Milburn G J 2001 Phys. Rev. A 64, 042315; Albeverio S and Fei S M 2001 J. Opt. B: Quantum 
Semiclasscal. Opt. 3, 223; Akhtarshenas S J 2005 J. Phys. A: Math. Gen. 38, 6777; Heydari H 2006 J. Phys. A: Math. Gen. 39, 15225

[5] Vidal G and Werner R F 2002 Phys. Rev. A 65, 032314

[6] Brody D C and Hughston L P 2001 J. Geom. Phys. 38, 19

[7] Wei T C and Goldbart P M 2003 Phys. Rev. A 68, 042307

[8] Bennett C H and Wiesner S J 1992 Phys. Rev. Lett. 69, 2881; Mattle K, Weinfurter H, Kwiat P G and Zeilinger A 1996 Phys. Rev. Lett. 76, 4656

[9] Bennett C H, Brassard G, Crépeau C, Jozsa R, Peres A and Wootters W K 1993 Phys. Rev. Lett. 70, 1895; Bouwmeester D, Pan J W, Daniell M, Weinfurter H and Zeilinger A 1997 Nature (London) 390, 575; Boschi D, Branca S, Martini F D, Hardy L and Popescu S 1998 Phys. Rev. Lett. 80, 1121

[10] Zukowski M, Zeilinger A, Horne M A and Ekert A K 1993 Phys. Rev. Lett. 71, 4287; Pan J W, Bouwmeester D, Weinfurter H and Zeilinger A 1998 Phys. Rev. Lett. 80, 3891

[11] Ekert A K 1991 Phys. Rev. Lett. 67, 661; Jennewein T, Simon C, Weihs G, Weinfurter H and Zeilinger A 2000 Phys. Rev. Lett. 84, 4729; Naik D S, Perterson C G, White A G, Berglund A J and Kwiat P G 2000 Phys. Rev. Lett. 84, 4733; Tittel W, Brendel J, Zbinden H and Gisin N 2000 Phys. Rev. Lett. 84, 4737

[12] Bennett C H, DiVincenzo D P, Smolin J A and Wootters W K 1996 Phys. Rev. A 54, 3824

[13] Horodecki M, Horodecki P and Horodecki R 1999 Phys. Rev. A 60, 1888

[14] Albeverio S, Fei S M and Yang W L 2002 Phys. Rev. A 66, 012301

[15] Zhou Z W and Guo G C 2000 Phys. Rev. A 61, 032108

[16] Ota Y, Yoshida M and Ohba I arxiv: 0704.1375

[17] Özdemir S K, Bartkiewicz K, Liu Y X and Miranowicz A 2007 Phys. Rev. A 76, 042325

[18] Grondalski J, Etlinger D M and James D F V 2002 Phys. Lett. A 300, 573

[19] Li M, Fei S M and Wang Z X 2008 Phys. Rev. A 78, 032332

[20] Chen K, Albeverio S and Fei S M 2005 Phys. Rev. Lett. 95, 040504

[21] Horodecki M and Horodecki P 1999 Phys. Rev. A 59, 4206

[22] Linden N, Popescu S and Smolin J A 2006 Phys. Rev. Lett. 97, 100502; Yu C S, Yi X X and Song H S 2007 Phys. Rev. A 75, 022332; Song W, Liu N L and Chen Z B 2007 Phys. Rev. A 76, 054303; Niset J and Cerf N J 2007 Phys. Rev. A 76, 042328; Ou Y C and Fan H 2007 Phys. Rev. A 76, 022320; Gour G 2007 Phys. Rev. A 76, 052320 
[23] Terhal B M and Vollbrecht K G H 2000 Phys. Rev. Lett. 85, 2625

[24] Rungta P and Caves C M 2003 Phys. Rev. A 67, 012307

[25] Chen K, Albeverio S and Fei S M 2006 Rep. Math. Phys. 58, 3

[26] Werner R F 1989 Phys. Rev. A 40, 4277 